\title{
Chebyshev Polynomial Expansions of Complete Elliptic Integrals
}

\author{
By W. J. Cody
}

1. Introduction. Numerical subroutines for machine computation of the complete elliptic integrals are usually based upon the well-known Gauss arithmeticgeometric mean process [1] or Hastings-type Chebyshev approximations [2], [3], partially because the power-series expansions for these functions converge so slowly. It is well known that the speed of convergence as well as the numerical stability of a power-series expansion can frequently be improved by converting it into a series of Chebyshev polynomials [4]. In this paper we present a number of such converted expansions for the complete elliptic integrals $K(k)$ and $E(k)$.

2. Power-Series Expansions for $K(k)$ and $E(k)$. The complete elliptic integral of the first kind is defined by

$$
K(k)=\int_{0}^{\pi / 2} \frac{d \phi}{\sqrt{ }\left(1-k^{2} \sin ^{2} \phi\right)}=\frac{\pi}{2}{ }_{2} F_{1}\left(\frac{1}{2}, \frac{1}{2} ; 1 ; m\right), \quad|k|<1,
$$

where $m=k^{2}$ and ${ }_{2} F_{1}(a, b ; c ; z)$ is Gauss' hypergeometric series [5]. Computationally this form is most useful for small $m$. For $m \approx 1$ there are two other useful expansions: the Legendre form [6]

$$
K(k)=\ln \frac{4}{\sqrt{ } \eta}{ }_{2} F_{1}\left(\frac{1}{2}, \frac{1}{2} ; 1 ; \eta\right)-K_{2}(\eta),
$$

and a modified Legendre form [3]

$$
K(k)=\frac{1}{2} \ln \frac{1}{\eta}{ }_{2} F_{1}\left(\frac{1}{2}, \frac{1}{2} ; 1 ; \eta\right)+\mathfrak{K}_{2}(\eta),
$$

where $K_{2}(\eta)$ and $\mathcal{K}_{2}(\eta)$ are infinite power series, and $\eta=1-m$.

The analogous equations for the complete elliptic integral of the second kind,

$$
\begin{aligned}
E(k) & =\int_{0}^{\pi / 2} \sqrt{ }\left(1-k^{2} \sin ^{2} \phi\right) d \phi, \quad|k| \leqq 1, \\
& =\frac{\pi}{2}{ }_{2} F_{1}\left(-\frac{1}{2}, \frac{1}{2} ; 1 ; m\right), \quad|k|<1,
\end{aligned}
$$

are

$$
E^{\prime}(k)=\ln \frac{4}{\sqrt{ } \eta} E_{1}^{\prime}(\eta)+E_{2}^{\prime}(\eta)
$$

for the Legendre form, and

$$
E(k)=\ln \frac{1}{\eta} \varepsilon_{1}(\eta)+\mathcal{E}_{2}(\eta)
$$

Received September 16, 1964. Work performed under the auspices of the U. S. Atomic Energy Commission. 
for the modified Legendre form, where $E_{1}, E_{2}, \varepsilon_{1}$, and $\varepsilon_{2}$ are all infinite power series.

3. Chebyshev Series Expansions. A sufficiently rapidly converging power series can be converted directly into a convergent expansion in shifted Chebyshev polynomials [7]

$$
f(x)=\sum_{n=0}^{\infty} a_{n} T_{n}^{*}(x), \quad 0 \leqq x \leqq 1,
$$

where

$$
T_{n}{ }^{*}(x)=\cos [n \arccos (2 x-1)],
$$

and the prime on the summation indicates that only half of the first term is used. All of the expansions of Section 2 are well behaved for values of the variable not greater than $\frac{1}{2}$. Further, the restricted domains $0 \leqq m \leqq \frac{1}{2}$ and $0 \leqq \eta \leqq \frac{1}{2}$ cover the full domain $0 \leqq k^{2} \leqq 1$, making it reasonable to convert the expansions into series of shifted Chebyshev polynomials in the variables $2 m$ and $2 \eta$.

There are two useful expansions corresponding to (1):

$$
K(k)=\left\{\begin{array}{l}
\sum_{n=0}^{\infty} a_{n} T_{n}{ }^{*}(2 m), \\
\pi \sum_{n=0}^{\infty} b_{n} T_{n}{ }^{*}(2 m),
\end{array} \quad 0 \leqq m \leqq \frac{1}{2},\right.
$$

where $(7 \mathrm{~b})$ results from conversion of ${ }_{2} F_{1}\left(\frac{1}{2}, \frac{1}{2} ; 1 ; m\right)$ and the coefficients of $(7 \mathrm{a})$ incorporate the multiplication by $\pi / 2$. The usefulness of $(7 \mathrm{~b})$ is pointed up by the expansions corresponding to (2) and (3), where the coefficients $b_{n}$ occur again:

$$
\text { (7c,d) } K(k)= \begin{cases}\ln \frac{16}{\eta} \sum_{n=0}^{\infty} b_{n} T_{n}{ }^{*}(2 \eta)-\sum_{n=0}^{\infty} c_{n} T_{n}{ }^{*}(2 \eta), & \\ \ln \frac{1}{\eta} \sum_{n=0}^{\infty} b_{n} T_{n}{ }^{*}(2 \eta)+\sum_{n=0}^{\infty} d_{n} T_{n}{ }^{*}(2 \eta), & 0 \leqq \eta \leqq \frac{1}{2} .\end{cases}
$$

The expansions corresponding to (4) and (6) are

$$
\text { (8a, b) } \quad E(k)=\left\{\begin{array}{l}
\sum_{n=0}^{\infty} p_{n} T_{n}^{*}(2 m), \quad 0 \leqq m \leqq \frac{1}{2}, \\
\eta \ln \frac{1}{\eta} \sum_{n=0}^{\infty} q_{n} T_{n}^{*}(2 \eta)+\sum_{n=0}^{\infty} r_{n} T_{n}^{*}(2 \eta), \quad 0 \leqq \eta \leqq \frac{1}{2} .
\end{array}\right.
$$

The peculiar form of (8b) is necessary to insure proper evaluation of $E(k)$ for $\eta=0\left(k^{2}=1\right)$ through the relation

$$
\lim _{\eta \rightarrow 0} \eta \ln \frac{1}{\eta}=0 .
$$

For most floating-point computers, the evaluation of $\ln (1 / \eta)$ is accurate for all $\eta$ except 0 . Then the expansion

$$
E(k)=\left\{\begin{array}{l}
\ln \frac{1}{\eta} \sum_{n=0}^{\infty} s_{n} T_{n}^{*}(2 \eta)+\sum_{n=0}^{\infty} r_{n} T_{n}^{*}(2 \eta), \quad 0<\eta \leqq \frac{1}{2}, \\
1, \quad \eta=0,
\end{array}\right.
$$

would be most useful. 
Table Ia

\begin{tabular}{|c|c|c|c|c|c|}
\hline \multirow{2}{*}{$\frac{i}{0}$} & \multicolumn{5}{|c|}{$a_{i}$} \\
\hline & 3.39777 & 24271 & 35543 & 97735 & 7142 \\
\hline 1 & .14003 & 09050 & 00041 & 35907 & 1349 \\
\hline 2 & .01333 & 84495 & 86727 & 63989 & 7455 \\
\hline 3 & .00157 & 90046 & 73690 & 96955 & 2093 \\
\hline 4 & .00020 & 66230 & 76906 & 06232 & 3139 \\
\hline 5 & .00002 & 86422 & 49149 & 18138 & 2405 \\
\hline 6 & & 41218 & 59188 & - 31631 & 7717 \\
\hline 7 & & 6089 & 55680 & 48805 & 5348 \\
\hline 8 & & 917 & 32241 & 42273 & 3436 \\
\hline 9 & & 140 & 26862 & 71140 & 9898 \\
\hline 10 & & 21 & 70501 & 60569 & 0423 \\
\hline 11 & & 3 & 39121 & 66692 & 5655 \\
\hline 12 & & & 53410 & 81137 & 5149 \\
\hline 13 & & & 8469 & 01565 & 7412 \\
\hline 14 & & & 1350 & 63745 & 3424 \\
\hline 15 & & & 216 & 47417 & 2743 \\
\hline 16 & & & 34 & 84651 & 1614 \\
\hline 17 & & & 5 & 63083 & 0298 \\
\hline 18 & & & & 91297 & 2705 \\
\hline 19 & & & & 14847 & 6531 \\
\hline 20 & & & & 2421 & 2461 \\
\hline 21 & & & & 395 & 8102 \\
\hline 22 & & & & 64 & 8490 \\
\hline 23 & & & & 10 & 6464 \\
\hline 24 & & & & 1 & 7511 \\
\hline 25 & & & & & 2885 \\
\hline 26 & & & & & 476 \\
\hline 27 & & & & & 79 \\
\hline 28 & & & & & 13 \\
\hline 29 & & & & & 2 \\
\hline
\end{tabular}


Table Ib

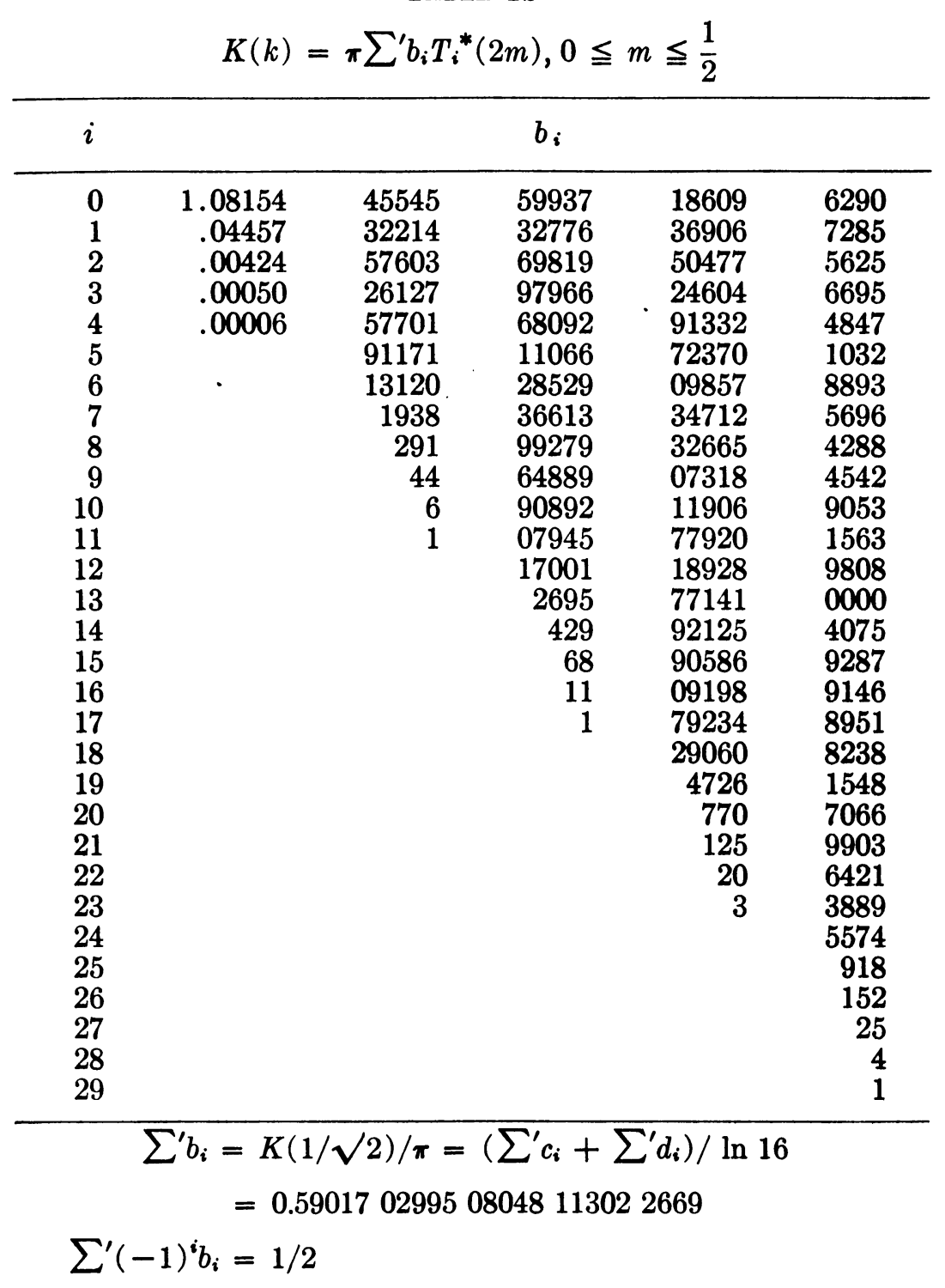


TABLE Ic

\begin{tabular}{|c|c|c|c|c|c|}
\hline$i$ & & & $c_{i}$ & & \\
\hline 0 & .17041 & 22621 & 30315 & 43155 & 1028 \\
\hline 1 & .09435 & 20461 & 78037 & 74863 & 9851 \\
\hline 2 & .01026 & 96473 & 11552 & 68891 & 5886 \\
\hline 3 & .00127 & 33175 & 37102 & 88162 & 3592 \\
\hline 4 & .00017 & 05201 & 42845 & 66669 & 0372 \\
\hline 5 & .00002 & 39656 & 59641 & 07412 & 6634 \\
\hline 6 & & 34804 & 63592 & - 88262 & 8676 \\
\hline 7 & & 5175 & 36666 & 20927 & 5519 \\
\hline 8 & & 783 & 38322 & 70517 & 9371 \\
\hline 9 & & 120 & 23636 & 50853 & 8075 \\
\hline 10 & & 18 & 66072 & 26752 & 2056 \\
\hline 11 & & 2 & 92265 & 83806 & 8435 \\
\hline 12 & & & 46124 & 04947 & 6578 \\
\hline 13 & & & 7326 & 05495 & 2348 \\
\hline 14 & & & 1170 & 05939 & 6522 \\
\hline 15 & & & 187 & 76803 & 7005 \\
\hline 16 & & & 30 & 25882 & 9099 \\
\hline 17 & & & 4 & 89424 & 3915 \\
\hline 18 & & & & 79422 & 5883 \\
\hline 19 & & & & 12926 & 3938 \\
\hline 20 & & & & 2109 & 3960 \\
\hline 21 & & & & 345 & 0459 \\
\hline 22 & & & & 56 & 5639 \\
\hline 23 & & & & 9 & 2910 \\
\hline 24 & & & & 1 & 5289 \\
\hline 25 & & & & & 2520 \\
\hline 26 & & & & & 416 \\
\hline 27 & & & & & 69 \\
\hline 28 & & & & & 11 \\
\hline 29 & & & & & 2 \\
\hline
\end{tabular}

$$
\begin{aligned}
\sum^{\prime} c_{i} & =0.191299718469738220630544 \\
\sum^{\prime}(-)^{i} c_{i} & =0
\end{aligned}
$$


Table Id

\begin{tabular}{|c|c|c|c|c|c|}
\hline$i$ & & & $d_{i}$ & & \\
\hline 0 & 2.82826 & 59724 & 42414 & 17553 & 4433 \\
\hline 1 & .02923 & 11648 & 80374 & 51558 & 6255 \\
\hline 2 & .00150 & 21000 & 07141 & 47287 & 6959 \\
\hline 3 & .00012 & 02210 & 38191 & 71382 & 0917 \\
\hline 4 & .00001 & 18334 & 83468 & 55949 & 4530 \\
\hline 5 & & 13123 & 39681 & 93150 & 8514 \\
\hline 6 & - & 1572 & 51910 & 15293 & 9813 \\
\hline 7 & & 198 & 92541 & 91411 & 8240 \\
\hline 8 & & 26 & 19269 & 85343 & 1502 \\
\hline 9 & & 3 & 55664 & 58182 & 5016 \\
\hline 10 & & & 49487 & 43009 & 3146 \\
\hline 11 & & & 7023 & 41195 & 9203 \\
\hline 12 & & & 1013 & 25621 & 3007 \\
\hline 13 & & & 148 & 21045 & 6753 \\
\hline 14 & & & 21 & 93542 & 3977 \\
\hline 15 & & & 3 & 27959 & 9077 \\
\hline 16 & & & & 49469 & 4914 \\
\hline 17 & & & & 7520 & 2573 \\
\hline 18 & & & & 1151 & 1240 \\
\hline 19 & & & & 177 & 2896 \\
\hline 20 & & & & 27 & 4564 \\
\hline 21 & & & & 4 & 2733 \\
\hline 22 & & & & & 6681 \\
\hline 23 & & & & & 1049 \\
\hline 24 & & & & & 165 \\
\hline 25 & & & & & 26 \\
\hline 26 & & & & & 4 \\
\hline 27 & & & & & 1 \\
\hline
\end{tabular}


Table IIa

$$
E(k)=\sum^{\prime} p_{i} T_{i}^{*}(2 m), 0 \leqq m \leqq \frac{1}{2}
$$

\begin{tabular}{|c|c|c|c|c|c|}
\hline$i$ & & & $p_{i}$ & & \\
\hline 0 & 2.92822 & 58504 & 05146 & 88299 & 9545 \\
\hline 1 & -.10983 & 85572 & 43451 & 91176 & 2083 \\
\hline 2 & -.00337 & 07796 & 33972 & 36148 & 2362 \\
\hline 3 & -.00023 & 53008 & 58731 & 36941 & 4039 \\
\hline 4 & -.00002 & 17641 & 44792 & 00668 & 4306 \\
\hline 5 & - & 23301 & 64928 & 43946 & 8235 \\
\hline 6 & - & 2729 & 92738 & 83921 & 9275 \\
\hline 7 & - & 339 & 98892 & 03979 & 0023 \\
\hline 8 & - & 44 & 25755 & 44400 & 3036 \\
\hline 9 & - & 5 & 95739 & 31848 & 8316 \\
\hline 10 & - & & 82323 & 46149 & 6100 \\
\hline 11 & - & & 11618 & 87697 & 1255 \\
\hline 12 & - & & 1668 & 57756 & 6166 \\
\hline 13 & - & & 243 & 13006 & 2812 \\
\hline 14 & - & & 35 & 86643 & 5645 \\
\hline 15 & - & & 5 & 34740 & 3815 \\
\hline 16 & - & & & 80463 & 4863 \\
\hline 17 & - & & & 12205 & 7159 \\
\hline 18 & - & & & 1864 & 7874 \\
\hline 19 & - & & & 286 & 7194 \\
\hline 20 & - & & & 44 & 3363 \\
\hline 21 & - & & & 6 & 8912 \\
\hline 22 & - & & & 1 & 0761 \\
\hline 23 & - & & & & 1687 \\
\hline 24 & - & & & & 266 \\
\hline 25 & - & & & & 42 \\
\hline 26 & - & & & & 7 \\
\hline 27 & - & & & & 1 \\
\hline
\end{tabular}


TABLE IIb

\begin{tabular}{|c|c|c|c|c|c|}
\hline$i$ & & & $q_{i}$ & & \\
\hline $\begin{array}{r}0 \\
1 \\
2 \\
3 \\
4 \\
5 \\
6 \\
7 \\
8 \\
9 \\
10 \\
11 \\
12 \\
13 \\
14 \\
15 \\
16 \\
17 \\
18 \\
19 \\
20 \\
21 \\
22 \\
23 \\
24 \\
25 \\
26 \\
27 \\
28 \\
29\end{array}$ & $\begin{array}{l}.56305 \\
.03478 \\
.00365 \\
.00045 \\
.00006 \\
.\end{array}$ & $\begin{array}{r}88879 \\
63815 \\
57095 \\
19021 \\
05772 \\
85266 \\
12400 \\
1846 \\
279 \\
42 \\
6 \\
1\end{array}$ & $\begin{array}{r}96356 \\
91808 \\
20277 \\
79315 \\
21314 \\
62411 \\
58661 \\
18799 \\
74114 \\
97300 \\
67435 \\
04600 \\
16516 \\
2624 \\
419 \\
67 \\
10 \\
1\end{array}$ & $\begin{array}{r}77758 \\
15239 \\
50596 \\
92957 \\
44649 \\
53353 \\
56426 \\
15787 \\
54148 \\
06565 \\
62997 \\
60020 \\
63047 \\
65105 \\
36432 \\
32345 \\
85276 \\
75591 \\
28502 \\
4639 \\
757 \\
123 \\
20 \\
3\end{array}$ & $\begin{array}{r}1788 \\
9162 \\
6041 \\
8839 \\
5014 \\
5347 \\
5501 \\
0220 \\
4018 \\
9237 \\
9643 \\
6540 \\
0207 \\
2908 \\
4946 \\
8184 \\
4985 \\
0866 \\
0895 \\
9668 \\
3400 \\
9073 \\
3160 \\
3376 \\
5493 \\
906 \\
150 \\
25 \\
4 \\
1\end{array}$ \\
\hline
\end{tabular}


TABLE IIb (Cont'd.)

\begin{tabular}{|c|c|c|c|c|c|}
\hline$i$ & & & $r_{i}$ & & \\
\hline 0 & 2.23448 & 68607 & 29039 & 00443 & 7906 \\
\hline 1 & .11961 & 91062 & 84828 & 73981 & 8781 \\
\hline 2 & .00252 & 62231 & 70143 & 06557 & 7453 \\
\hline 3 & .00016 & 38806 & 47349 & 18297 & 3709 \\
\hline 4 & .00001 & 47258 & 39374 & 78249 & 4237 \\
\hline 5 & & 15529 & 49525 & 56853 & 6576 \\
\hline 6 & & 1802 & 70038 & 62290 & 4853 \\
\hline 7 & & 223 & 13086 & 14184 & 5666 \\
\hline 8 & & 28 & 91801 & 65272 & 7708 \\
\hline 9 & & 3 & 87974 & 70162 & 2529 \\
\hline 10 & & & 53475 & 80157 & 8024 \\
\hline 11 & & & 7531 & 97267 & 3412 \\
\hline 12 & & & 1079 & 85373 & 6609 \\
\hline 13 & & & 157 & 12789 & 2994 \\
\hline 14 & & & 23 & 15222 & 5698 \\
\hline 15 & & & 3 & 44834 & 7385 \\
\hline 16 & & & & 51842 & 8847 \\
\hline 17 & & & & 7858 & 2084 \\
\hline 18 & & & & 1199 & 7722 \\
\hline 19 & & & & 184 & 3607 \\
\hline 20 & & & & 28 & 4931 \\
\hline 21 & & & & 4 & 4265 \\
\hline 22 & & & & & 6909 \\
\hline 23 & & & & & 1083 \\
\hline 24 & & & & & 170 \\
\hline 25 & & & & & 27 \\
\hline 26 & & & & & 4 \\
\hline 27 & & & & & 1 \\
\hline
\end{tabular}


TABLE IIC

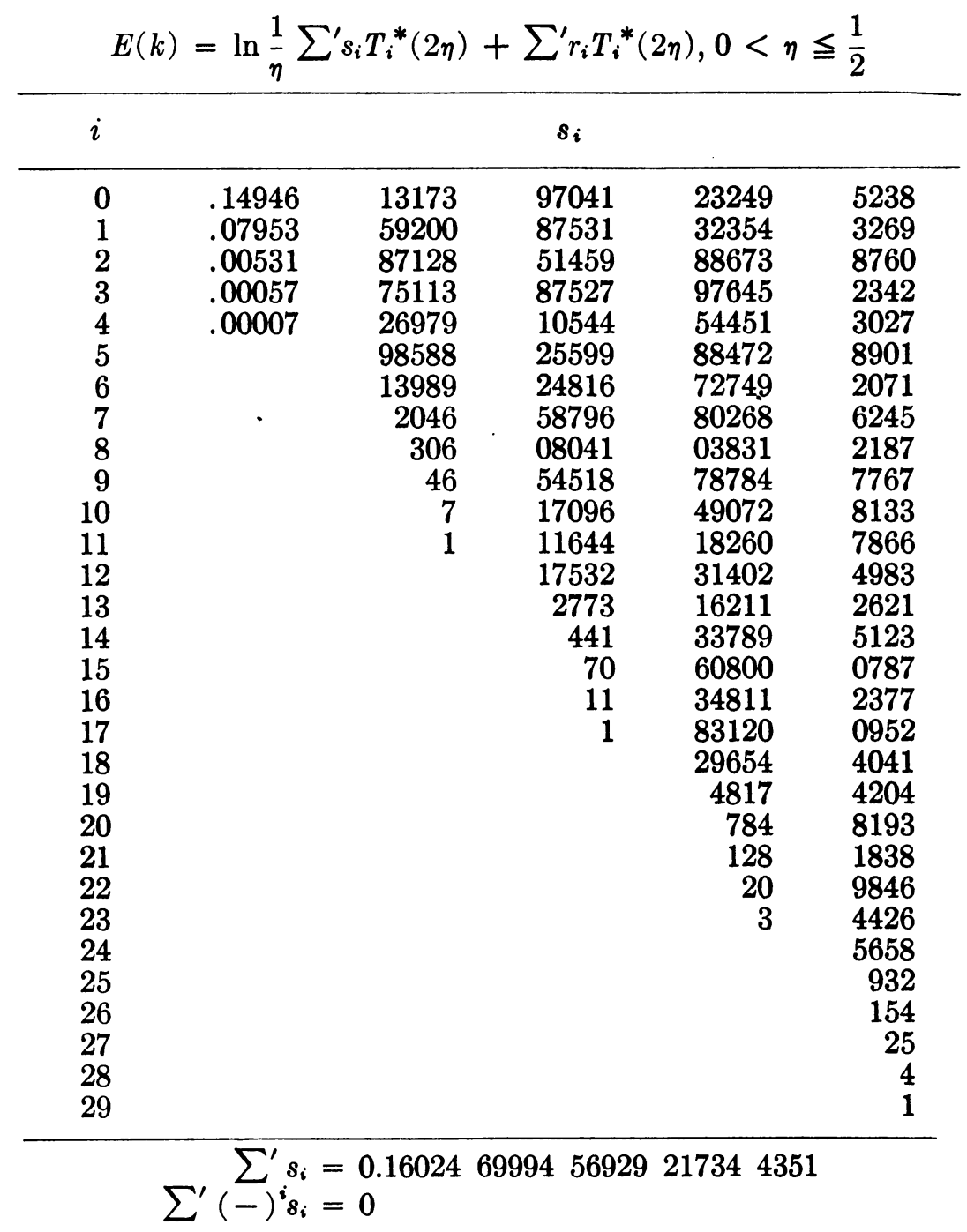


4. Results. The coefficients for the series (7) and (8) were computed on a CDC 3600 in 25-decimal arithmetic. The program for the conversion of the power series to a series of Chebyshev polynomials was a revision of a program originally written by Dr. H. C. Thacher of Argonne National Laboratory. The coefficients, together with theoretical sums, are given in Tables I and II.

All expansions were checked by using the tabulated coefficients to compute complete elliptic integrals for 2000 random arguments in 25-decimal arithmetic. Results were compared against a 25-decimal routine based on the Gauss arithmetic-geometric mean process [1]. In all cases, the maximal errors encountered were within limits imposed by round-off.

Argonne National Laboratory

Argonne, Illinois

1. M. Abramowitz \& I. A. Stegun, (Eds.), Handbook of Mathematical Functions, National Bureau of Standards, Applied Mathematics Series, Vol. 55, Washington, D. C., 1964.

2. C. Hastings, JR., A pproximations for Digital Computers, Princeton Univ. Press, Princeton, N. J., 1955. MR 16, 963.

3. W. CoDY, JR., "Chebyshev approximations for the complete elliptic integrals $K$ and E," Math. Comp., v. 19, 1965, pp. 105-112.

4. C. W. Clenshaw, Chebyshev Series for Mathematical Functions, National Physical Laboratory Mathematical Tables, Vol. 5, Department of Scientific and Industrial Research, Her Majesty's Stationery Office, London, 1962. MR 26 *362.

5. A. ERdelyi ET AL., Higher Transcendental Functions, Vol. II, McGraw-Hill, New York, 1953. MR 15, 419.

6. J. R. AIREy, "Toroidal functions and the complete elliptic integrals," Philos. Mag., v. 19,1935 , pp. $177-188$.

7. H. C. ThaCher, JR., "Conversion of a power series to a series of Chebyshev polynomials," Comm. ACM, v. 7, 1964, pp. 181-182. 